\title{
Breast cancer progression, prognosis and prospective outcomes
}

\section{Editorial}

Breast Cancer is the most common cancer globally and takes more feminine lives than any other cancer, the global efforts did have some effect and the progression of breast cancer has gone down slightly on an average around the world in principle and mostly due to progress in detection methodology and awareness and this perception is in developed world. The story is totally different in the developing world. Breast cancer is a contrasting disease with an asymmetric morphaology, molecular features, which makes it atypical in response to therapies. Conventionally, prognostic markers in breast cancer is based on the clinic-pathological parameters and detached molecular markers, but on the other hand breast cancer prognosis behaves to some extent asymmetrically in different ethnic groups; although this is a debatable topic, but this situation still exists.

According to the work done in my lab the most significant issues is the average age at which the breast cancer effect the women of east and west, there is a clear difference of 10years as the average age of eastern women is 50years and western female gets at 60years and this difference of 10year is being carried by the migrant women in the west as well and many studies should this difference is purely genetic and not environmental. Based these studies and advocacy group the age of mammography has been reduced to 10years for eastern origin women. Once the breast cancer has been diagnosed the next step is prognosis. Family history does play a different role in prognosis according to a contemporarily published study where an accumulated number of ER negative \& PR negative breast cancer was acclaimed among younger Spanish women who have a family account of the disease. ${ }^{1}$ The established/routinely used prognostic markers which are being used by some of the highly respected institutes are ER, PR, Her2, p53, CD31, Ki-67/PCNA. Trastuzumab is being offered to Her2 positive patients who will benefit with this monoclonal drug or in other words Her-2 is being performed on a selective group of patients. Whereas ER, PR, p53, CD31, Ki-67/PCNA are being performed on almost every breast cancer patient.

Histological, the majority of breast cancers (65\% to $80 \%)$ belong to an ascetic subtype, invasive ductal carcinoma. This communicatively contours the use of type as a prognostic mediator. ${ }^{2}$ Therefore, assessment of tumor behavior for any breast cancer case has been based on deltoid parameters: tumor size, lymph node condition, and histological grade. Tumor size is a good prognostic marker for metastasis or otherwise in lymph node negative patients, although patients with small tumors $(<1 \mathrm{~cm})$ after the surgical removal are not offered any further treatment, have algorithmically a $12 \%$ adventitious of periodicity. ${ }^{3,4}$ Lymph node status is still appraised the best prognostic indicator of relapse, but with the commencement of the drugs like tamoxifen and trastuzumab, a more molecular characterization of the tumor is in great demand. There are about 54 drugs which have been approved by the Food and Drug Administration for treatment; so the oncologist thus requires some kind of may be accurate advocacy about the molecular characteristic of the tumor to deal with some precision.

\author{
Volume 4 Issue 6 - 2017
}

\begin{abstract}
Syed A Aziz
Department of Pathology, University of Ottawa, Canada

Correspondence: Syed A Aziz, Scientist Health Canada and Adjunct Professor, Department of Pathology, University of Ottawa, Canada, Email syed.a.aziz@hc-sc.gc.ca
\end{abstract}

Received: June 09, 2017 | Published: June 21, 2017

Established clinical approaches in patients with tumors are based on the adherence to the determination of chemotherapy depending on the histopathological account of the tumor as well as its organ of origin. Molecular approaches of neoplasia encompass an ample enumerate of genetic abnormalities as well as the existence of circulating tumor cells (CTCs) in blood, which proceeds to a poor prognosis for the patient. ${ }^{5}$ Biomarkers for personalized oncology are applied mainly in molecular diagnostics of chronic myeloid leukemia, colon, breast and lung cancer, and more recently in melanoma. They are certainly applied in the assessment of the advantages that can be achieved through targeted therapy or in the evaluation of toxic effects of the chemotherapeutic used in the therapy.

More recently, a battery of four prognostic markers (ER, PR, HER2 and Ki67) has been shown to have a high prognostic impact which could be similar to that of gene expression assays. ${ }^{6}$ Some other markers, like serine protease urokinase-type plasminogen activator (uPA) and its inhibitor (plasminogen activator inhibitor type-1;PAI-1) have reached the evidence level by where it can be judged by the American Society for Clinical Oncology as acceptable for clinical use in patients with newly diagnosed node negative breast cancer using an ELISA assay. These assays, although are quantitative, and limited to the use of fresh/frozen tissues. In the case of uPA/PAI-1, a miserly of $300 \mathrm{mg}$ of fresh/ frozen breast cancer tissue is needed. Given the fringed availability of frozen tissues in the clinical setting, this heightens a doubt about its exercise in routine endeavor. Another prognostic which has shown the potential is VE-cadherin especially in metastatic breast cancer and has the capability to be an independent prognostic factor for both progression-free survival and overall survival. ${ }^{7}$ As a matter of fact all the medical jargon is pretty useless if the prospective outcome is not better for an individual patients but for the sack of argument all the work has been under way to for the establishment of the better outcome. There are many things which can affect the outcome of any disease either positive or negative. The awareness and understanding of a disease (probably more is required for cancer and/or breast cancer) based on the fact that women in general are little more careless about their health. The unfortunate part is with all the awareness and/or understanding if the standard medical facilities are not available or affordable than things could go ugly. 


\section{Acknowledgements}

None.

\section{Conflict of interest}

The author declares no conflict of interest.

\section{References}

1. Jiang X, Castelao JE, Chavez-Uribe E, et al. Family history and breast cancer hormone receptor status in a Spanish cohort. PLoS One. 2012;7(1):e29459.

2. Elston CW, Ellis IO, Pinder SE. Pathological prognostic factors in breast cancer. Crit Rev Oncol Hematol. 1999;31(3): 209-223.

3. Fisher B, Dignam J, Tan-Chiu E, et al. Prognosis and treatment of patients with breast tumors of one centimeter or less and negative axillary lymph nodes. J Natl Cancer Inst. 2001;93(2):112-120.
4. Rosen PP, Groshen S, Saigo PE, et al. Pathological prognostic factors in stage I (T1N0M0) and stage II (T1N1M0) breast carcinoma: a study of 644 patients with edian follow-up of 18 years. J Clin Oncol. 1989;7(9):12391251 .

5. Iwata H, Masuda $\mathrm{N}$, Yamamoto $\mathrm{D}$, et al. Circulating tumor cells as a prognostic marker for efficacy in the randomized phase III JO21095 trial in Japanese patients with HER2-negative metastatic breast cancer. Breast Cancer Res Treat. 2017;162(3):501-510.

6. Cuzick J, Dowsett M, Pineda S, et al. Prognostic value of a combined estrogen receptor, progesterone receptor, $\mathrm{Ki}-67$, and human epidermal growth factor receptor 2 immunohistochemical score and comparison with the Genomic Health recurrence score in early breast cancer. J Clin Oncol. 2011;29(32):4273-4278.

7. Rochefort P, Chabaud S, Pierga JY, et al. Soluble VE-cadherin in metastatic breast cancer: an independent prognostic factor for both progression-free survival and overall survival. Br J Cancer. 2017;116(3):356-361. 